\title{
Laser-Induced Breakdown Spectroscopy for Studying the Electrochemical Impact of Porosity Variations in Composite Electrode Materials
}

\author{
P. Smyrek ${ }^{1,2}$, Y. Zheng ${ }^{1}$, J.-H. Rakebrandt ${ }^{1}$, \\ H.J. Seifert ${ }^{1}$, and W. Pfleging ${ }^{1,2}$
}

\author{
${ }^{1}$ Institute for Applied Materials (IAM-AWP) \\ ${ }^{2}$ Karlsruhe Nano Micro Facility (KNMF) \\ Karlsruhe Institute of Technology, P.O. Box 3640, \\ 76021 Karlsruhe, Germany
}

\begin{abstract}
The porosity in composite electrode materials can vary on micro- and nanometer scale and has a great impact on electrochemical performance in lithium-ion cells. Liquid electrolyte has to penetrate into the entire porous electrodes in order to enable lithium-ion diffusion. For studying the electrochemical impact of porosity variations in composite lithium-nickel-manganese-cobalt-oxide thick films $\left(\mathrm{Li}\left(\mathrm{Ni}_{1 / 3} \mathrm{Mn}_{1 / 3} \mathrm{Co}_{1 / 3}\right) \mathrm{O}_{2}, \quad \mathrm{NMC}\right), \quad$ laser-induced breakdown spectroscopy (LIBS) was applied. A rapid chemical screening of the complete electrode after electrochemical cycling and cell degradation was performed. This rather new technological approach was used to obtain post-mortem critical information about surface and bulk phenomena that define and control the performance of lithium-ion batteries. The influence of porosity variations along NMC electrode surfaces was studied regarding capacity retention, life-time, and lithium distribution. For this purpose, different geometrical arrangements of porosity distribution were generated by embossing. Using LIBS, elemental mapping of lithium was obtained with a lateral resolution of $100 \mu \mathrm{m}$. A correlation between porosity distribution, cell degradation and local lithium plating could be identified.
\end{abstract}

Keywords - Laser-induced breakdown spectroscopy; lithium-ion battery; lithium nickel manganese cobalt oxide; cathode; porosity

\section{INTRODUCTION}

The precise control of porosity and pore size distribution in manufacturing of composite electrode materials is a critical task, which finally influences the electrochemical performance of lithium-ion cells [1]. In addition, the porosity has an impact on electrical conductivity and strength of coating adhesion between the composite material and the current collector [2-4]. Furthermore, with reducing of porosity in electrodes an increasing of volumetric energy is achieved, which is quite important for application such as portable systems or electromobility purposed with a limited space [1]. Nevertheless, electrodes with a low porosity may hinder the lithium-ion migration [2] and the electrolyte penetration processes become more challenging, especially for high electrode thicknesses in high energy applications [5] Therefore, the development of electrode architectures is quite crucial in order to provide advanced electrochemical performances in lithium-ion batteries. One possible approach for improving the battery performance is the generation of three-dimensional (3D) architectures in electrode materials, e.g. by introducing free-standing micro-pillars or micro-capillary structures. It could be clearly demonstrated, that lithium-ion cells with laser generated artificial porosities achieve a significant improvement in lithium-ion diffusion kinetics and a homogenous and rapid liquid electrolyte wetting could be achieved $[6,7]$.

It is obvious, that for next generation batteries, an optimized cell architecture is a critical task, which has to be investigated in detail. As described above, the porosity inside of composite electrodes is a main design parameter. It is assumed that the porosity and its distribution along the entire electrode has an impact on cell performance and cell degradation. This in turn means that we will expect a local change in stoichiometry during cell operation. An appropriate and rather new analytical method for such studies is laserinduced breakdown spectroscopy (LIBS), which offers the possibility to characterize very fast the elemental composition of the entire electrode. Furthermore, LIBS is capable of performing direct 3D analysis with micrometric [8] or nanometric resolution [9]. Only one single laser pulse is required in order to generate a laser-induced plasma and the achieved chemical information can be directly correlated with a local State-of-Health (SOH). Characterization methods such as bulk analysis, elemental depth profiling, elemental mapping as well as layer-by-layer analysis can be performed at atmospheric pressure condition [10, 11].

In this work we investigate post-mortem the lithium distribution in thick film NMC electrodes by using LIBS as function of porosity variations within the electrodes. For this purpose, elemental mapping of lithium was performed by using a lateral resolution of $100 \mu \mathrm{m}$. To our knowledge, there is no comparable study in which electrodes with varying porosities were quantitatively analyzed post-mortem by LIBS.

\section{EXPERIMENTAL}

\section{Adjustment of porosity variations in NMC thick film} electrodes by embossing

Lithium nickel manganese cobalt oxide (NMC) composite cathodes were manufactured with a film thickness of $140 \mu \mathrm{m}$. The weight proportion was set to $90 \mathrm{wt} \%$ of NMC $(\mathrm{Ni}: \mathrm{Mn}: \mathrm{Co}=1: 1: 1, \quad$ MTI Corporation, USA), $5 \mathrm{wt} \%$ of conductive agent (Timcal Super C65, MTI Corporation, USA), 
and $5 \mathrm{wt} \%$ polyvinylidene fluoride (PVDF) binder (MTI Corporation, USA). N-Methyl-2-pyrrolidone (NMP) (BASF, Germany) was used as solvent. After drying, the porosity within the cathodes was locally varied by embossing (figure 1). Porosities in the range of $34 \%-55 \%$ were achieved.

\section{Porosity distribution in NMC thick film electrodes}

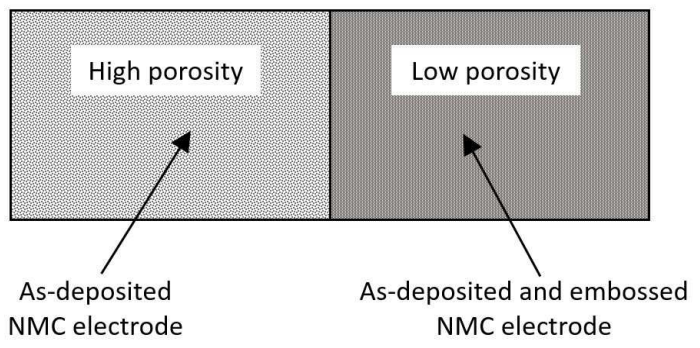

Figure 1. Schematic view of porosity distribution within a model NMC thick film electrode.

\section{Cell assembly and electrochemical characterization}

The principle set-up of the electrochemical cell is described elsewhere [12]. Briefly, all cells were assembled using the Swagelok ${ }^{\circledR}$ cell design, consisting of two plungers (stainless steel), three stainless steel Swagelok ${ }^{\circledR}$ components (product number: SS-12M0-6), one spring (stainless steel), four sealing rings (polytetrafluoroethylene, PTFE), and a $25 \mu \mathrm{m}$ thick polyamide foil (PI-foil) which acts as an isolator. Metallic lithium foil with a thickness of $350 \mu \mathrm{m}$ (Sigma Aldrich Chemistry, USA) was used as reference electrode. The separator material was a glass micro-fiber (GF/A filter) with a thickness of $260 \mu \mathrm{m}$ (Whatman Company). Ethylene carbonate and dimethyl carbonate with one molar lithium hexafluorophosphate conducting salt (EC:DMC 1:1, 1M $\mathrm{LiPF}_{6}, \mathrm{BASF}$, Germany) was used as electrolyte.

All battery tests were carried out using a BT2000 battery cycler (Arbin Instruments, USA). In a first approach, electrochemical formation was applied for three cycles with a C-rate of $\mathrm{C} / 10$ (charging and discharging). In a second approach, the cells were electrochemically cycled using a Crate for charging and discharging of $\mathrm{C} / 2$ and $1 \mathrm{C}$. The measured cells suffered from a spontaneous cell failure for cycles in the range of $70-147$.

\section{Laser-Induced Breakdown Spectroscopy (LIBS)}

The experimental setup of LIBS (SN013, Secopta Analytics GmbH, Germany) used for post-mortem investigation of NMC thick films is described elsewhere [13]. A pulsed mode-locked diode pumped solid state Nd:YAG laser (DPSS) operating at a wavelength of $1064 \mathrm{~nm}$ is focused onto an electrode surface and generates a laser-induced plasma. Laser power densities of $25.47 \mathrm{GW} / \mathrm{cm}^{2}$ could be achieved at the electrode surface. The plasma light of each laser pulse is focused onto an optical fiber which is attached to a Czerny-Turner spectrometer equipped with a ruled grating of 1200 grooves $/ \mathrm{mm}$ blazed at $250 \mathrm{~nm}$. The output end of the spectrometer is coupled with a back-thinned charge-coupled detector (CCD) including an electronic shutter (Hamamatsu S11155). The detector covers spectral ranges of $229 \mathrm{~nm}-498 \mathrm{~nm}$ and $569 \mathrm{~nm}-792 \mathrm{~nm}$ and was used to detect the dispersed light from the generated plasma.

\section{RESULTS AND DISCUSSION}

\section{Post-mortem LIBS characterization of NMC thick films} with varying porosity

For post-mortem identification of chemical inhomogeneities on electrode surfaces with varying porosity, LIBS was performed. Elemental mapping of lithium is appropriate in order to investigate whether lithium-ions could be deintercalated homogeneously from the cathode surface. A LIBS dot matrix with a lateral resolution of $100 \mu \mathrm{m}$ was applied. The measurement area at the cathode surface was adjusted to $13 \mathrm{~mm} \times 13 \mathrm{~mm}$, which corresponds to $131 \times 131=17131$ measurement locations.

Based on preliminary studies, it could be already shown that LIBS is a suitable chemical analysis tool in order to precisely measure lithium distributions along electrode surfaces after electrochemical cycling. As depicted in figure 2, NMC electrodes with homogenous porosity distribution were post-mortem characterized by LIBS. Figure 2 (a) and (b) demonstrate a lithium mapping along a fully lithiated NMC electrode directly after coating (a) and after electrochemical formation (b). This result clearly indicates that no difference in lithium distribution could be detected. After electrochemical formation (3 cycles using a C-rate of $\mathrm{C} / 10$ for charging/discharging [12]) the NMC electrodes show a high homogeneity regarding lithium distribution. The lithium distribution in NMC electrodes (no porosity variation) after cell operation at higher $\mathrm{C}$-rates (100 cycles using a C-rate of $\mathrm{C} / 2$ for charging/discharging [12]), was also measured by LIBS (figure 2 (c)). However, the cell could not reach the aimed 100 cycles due to spontaneous cell failure at cycle number 93. After performing an elemental mapping, a locally increased lithium concentration (position $\mathrm{X}=4.8 \mathrm{~mm}$ and $\mathrm{Y}=$ $7.2 \mathrm{~mm}$ ) could be observed. A possible cause for this type of degradation might be an insufficient liquid electrolyte wetting of the electrode caused by a material inhomogeneity which in turn might induce a local change in porosity. Good wetting properties are quite necessary for adequate electrochemical cyclability, especially for low porosity NMC thick films at high charging/discharging C-rates and for the use of thick film electrodes with respect to high energy applications.

In current research, different geometrical arrangements of porosity distribution were generated by embossing. For all NMC electrodes, the lithium distribution was investigated layer-by-layer using LIBS. We selected layer number five for the following discussion. In a first approach a model NMC electrode, as described in figure 1, was characterized postmortem by LIBS after cell failure (figure 3 (a)). The elemental mapping of lithium clearly demonstrates that the NMC electrode shows an inhomogeneous lithium distribution along the electrode surface which correlates to the porosity 
distribution in figure 1. Areas with increased lithium concentrations are correlated to areas with less porosity. The cell failure was obviously induced by local lithium plating which could be detected post-mortem in an area between low and high porosity (figure 3 (a)).
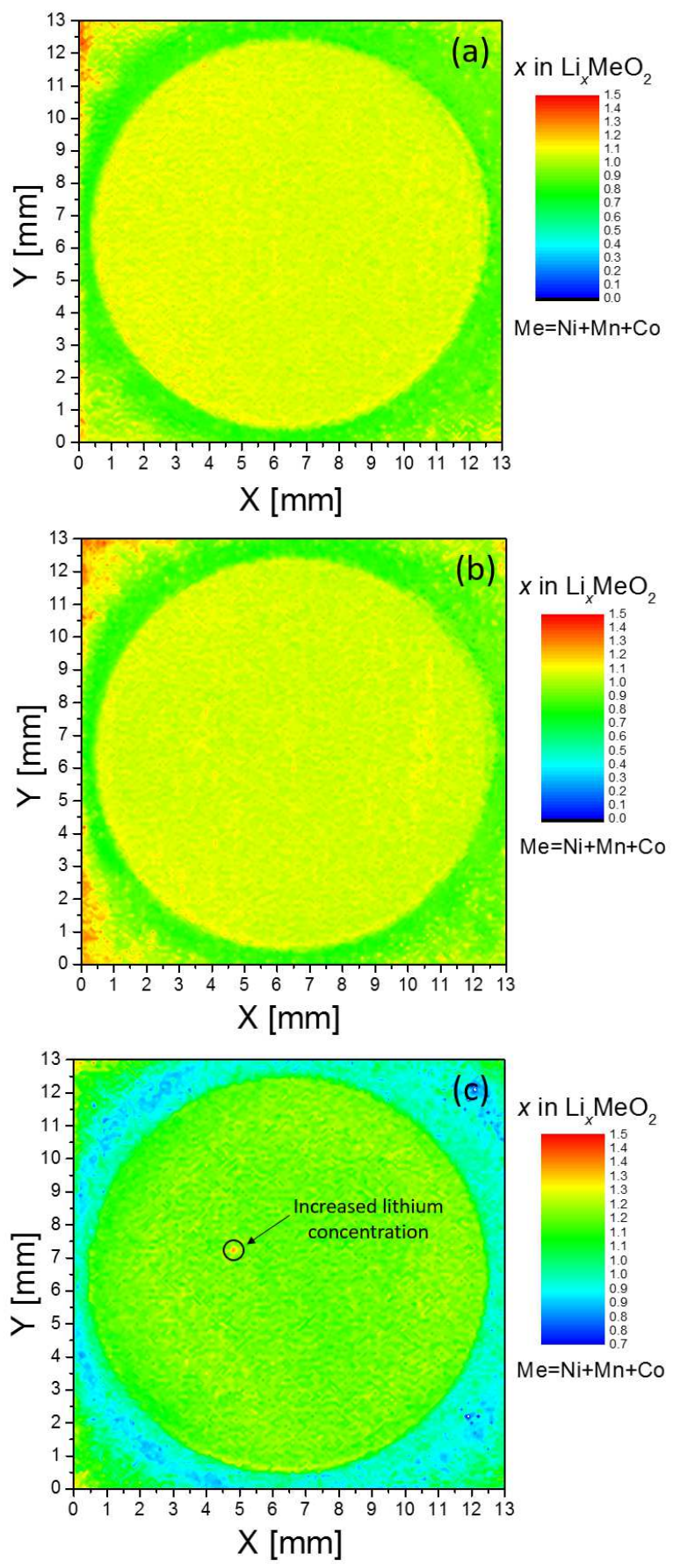

Figure 2. LIBS elemental mapping (lithium) of NMC electrodes with homogenous and low porosity as function of SoH: (a) lithium distribution after electrode coating, (b) after formation (3 cycles with $\mathrm{C} / 10-\mathrm{C} / 10$ ) and (c) after electrochemical cycling (93 cycles with $\mathrm{C} / 2-\mathrm{C} / 2)$. The elemental mappings represent the lithium distribution $\mathrm{x}$ in $\mathrm{Li}_{\mathrm{x}}\left(\mathrm{Ni}_{1 / 3} \mathrm{Mn}_{1 / 3} \mathrm{Co}_{1 / 3}\right) \mathrm{O}_{2}[12]$.
It is obvious that the electrode footprint area has an impact on cell lifetime, rate capability and cell degradation processes. Electrode surfaces, which indicate a variation of porosity and therefore an inhomogeneous lithium distribution, could lead to different electrical current densities. During battery operation, this aspect in turn would force the chemically driven aging process.

In a second approach, local embossing was used in order to adjust different porosity regions within the electrode (figure 3 (b)). Similar to the result of figure 3 (a), an increase of lithium content, which can be assigned to local lithium plating, could be detected at the area between high and low porosity. The post-mortem analysis was performed after spontaneous cell failure at cycle number 147 . As already mentioned, a well-defined porosity is quite necessary for an adequate electrochemical cyclability, especially for NMC thick films operating at high charging and discharging C-rates.
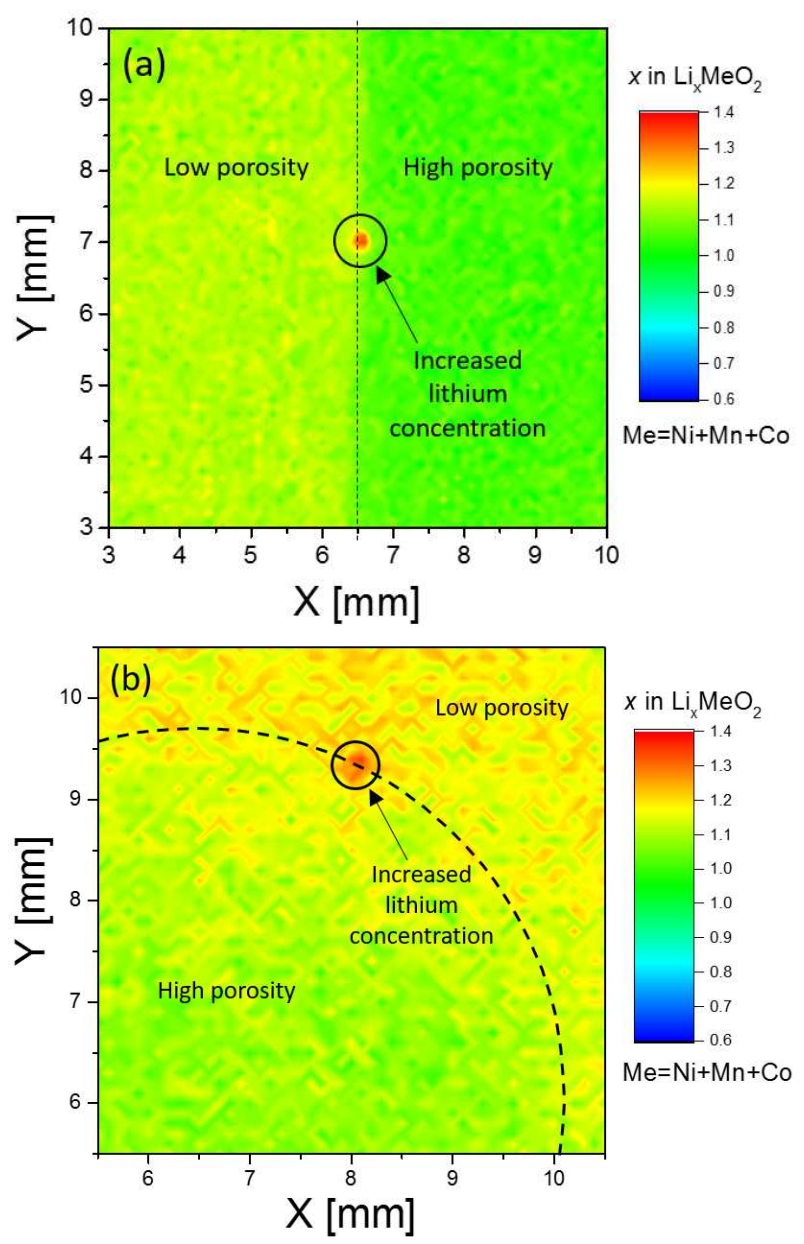

Figure 3. LIBS elemental mapping (lithium) of NMC thick film electrodes with varying porosity: (a) lithium distribution in NMC thick films with straight boundary between regions of different porosity, (b) lithium distribution in NMC thick films with curved boundary between regions of different porosity. The elemental mappings illustrate the lithium distribution $\mathrm{x}$ in $\mathrm{Li}_{\mathrm{x}}\left(\mathrm{Ni}_{1 / 3} \mathrm{Mn}_{1 / 3} \mathrm{Co}_{1 / 3}\right) \mathrm{O}_{2}$. 


\section{CONCLUSION}

Lithium nickel manganese cobalt oxide composite cathodes were tape-casted onto $20 \mu \mathrm{m}$ thick aluminum foils and film thicknesses of $140 \mu \mathrm{m}$ could be achieved. For all cathodes a variation of porosity distributions was generated by embossing. Laser-induced breakdown spectroscopy was applied for achieving elemental mappings of lithium after electrochemical cycling. A correlation of lithium distribution and porosity could be detected after cell failure. A high porosity leads to an increase of lithium content. Furthermore, lithium plating was observed in the area between low and high porosity. Electrode surfaces with porosity variation will induce an inhomogeneous lithium distribution. Furthermore, this will have an impact on local electrical current densities which in turn will enhance the chemically-driven aging process during battery operation.

\section{ACKNOWLEDGMENT}

We are grateful to our colleague $\mathrm{H}$. Besser for his technical assistance during laser materials processing. This project has received funding from the European Union's Horizon 2020 research and innovation programme under the Marie Sklodowska-Curie grant agreement no. 644971. Finally, the support for laser materials processing by the Karlsruhe Nano Micro Facility (KNMF, http://www.knmf.kit.edu/) a Helmholtz research infrastructure at the Karlsruhe Institute of Technology (KIT) is gratefully acknowledged.

\section{REFERENCES}

[1] Meyer, C., Bockholt, H., Haselrieder, W. and Kwade, A., "Characterization of the calendering process for compaction of electrodes for lithium-ion batteries," J. Power Sources, In Press, Accepted Manuscript, 2017.

[2] Zheng, H. H., Tan, L., Liu, G., Song, X. Y. and Battaglia, V. S., "Calendering effects on the physical and electrochemical properties of $\mathrm{Li}\left[\mathrm{Ni}_{1 / 3} \mathrm{Mn}_{1 / 3} \mathrm{Co}_{1 / 3}\right] \mathrm{O}_{2}$ cathode," J. Power Sources, vol. 208, pp. 52-57, 2012.

[3] Du, Z., Dunlap, R. A. and Obrovac, M. N., "High energy density calendered Si alloy/graphite anodes," J. Electrochem. Soc., vol. 161, pp. A1698-A1705, 2014.

[4] Zheng, H., Liu, G., Song, X., Ridgway, P., Xun, S. and Battaglia, V. S., "Cathode performance as a function of inactive material and void fractions,” J. Electrochem. Soc., vol. 157, pp. A1060-A1066, 2010.

[5] Singh, M., Kaiser, J. and Hahn, H., "Thick electrodes for high energy lithium ion batteries," J. Electrochem. Soc., vol. 162, pp. A1196-A1201, 2015.

[6] Pfleging, W. and Proell, J., "A new approach for rapid electrolyte wetting in tape cast electrodes for lithium-ion batteries," J. Mater. Chem. A, vol. 2, pp. 14918-14926, 2014.

[7] Mangang, M., Seifert, H. J. and Pfleging, W. "Influence of laser pulse duration on the electrochemical performance of laser structured LiFePO4 composite electrodes," J. Power Sources, vol. 304, pp. 24-32, 2016.
[8] Vadillo, J.M. and Laserna, J.J., "Laser-induced plasma spectrometry: truly a surface analytical tool," Spectrochim. Acta, Part B, vol. 59(2), pp. 147-161, 2004.

[9] Vadillo, J.M., Garcia, C.C., Palanco, S. and Laserna, J.J. "Nanometric range depth-resolved analysis of coated-steels using laser-induced breakdown spectrometry with a $308 \mathrm{~nm}$ collimated beam," J. Anal. At. Spectrom., vol. 13(8), pp. 793-797, 1998.

[10] Hou, H., Cheng, L., Richardson, T., Chen, G., Doeff, M., Zheng, R., Russo, R. and Zorba, V., "Threedimensional elemental imaging of Liion solid-state electrolytes using fs-laser induced breakdown spectroscopy (LIBS)," J. Anal. At. Spectrom., vol. 30, pp. 2295-2302, 2015

[11] Zorba, V., Syzdek, J., Mao, X.L., Russo, R.E. and Kostecki, R., "Ultrafast laser induced breakdown spectroscopy of electrode/electrolyte interfaces," Appl. Phys. Lett., vol. 100(23), pp. 234101 - 234105, 2012.

[12] Smyrek, P., Zheng, Y., Rakebrandt, J.H., Seifert, H.J. and Pfleging, W., "Investigation of micro-structured $\mathrm{Li}\left(\mathrm{Ni}_{1 / 3} \mathrm{Mn}_{1 / 3} \mathrm{Co} 1 / 3\right) \mathrm{O}_{2}$ cathodes by laser-induced breakdown spectroscopy," Proc. SPIE 10092, pp. 0S10S7, 2017.

[13] Smyrek, P., Proell, J., Seifert, H.J. and Pfleging, W., "Laser-induced breakdown spectroscopy of laser-structured $\mathrm{Li}(\mathrm{NiMnCo}) \mathrm{O}_{2}$ electrodes for lithium-ion batteries," J. Electrochem. Soc., vol. 163, pp. A19-A26, 2015 